\title{
Vers un modèle quantique pour la méditation
}

\author{
François Dubois ${ }^{1}$ et Christian Miquel ${ }^{2}$
}

15 novembre $2014^{3}$

\begin{abstract}
Résumé
Nous étudions les états méditatifs de l'être humain à partir du cadre conceptuel fourni par l'hypothèse fractaquantique : de façon analogue à un atome, l'Homme peut à partir de son état fondamental " tranquille » explorer divers états de plus haute énergie comme l'état amoureux ou l'état mystique. Nous cherchons ensuite quels états d'énergie sont explorés lors de la méditation : s'agit-il de la structure « hyperfine » de son état fondamental ou bien s'agit-il d'états d'extase amoureuse voire d'un état mystique de très haute énergie? Nous illustrons ces hypothèses à partir de l'expérience d'une part des grandes traditions mystiques comme l'hindouisme ou le bouddhisme et d'autre part des sciences cognitives contemporaines. De plus, la physique quantique indique que toute interaction entre niveaux d'énergie est médiatisée par un boson d'échange. Nous posons donc la question d'identifier la nature de ce boson d'interaction qui relie les divers niveaux d'énergie de l'être humain.
\end{abstract}

\section{Towards a quantum model for meditation}

\begin{abstract}
We study the meditative states of human beings from the conceptual framework provided by the fractaquantum hypothesis : analogously to an atom, Man can from his "quiet" base state explores various states of higher energy as loving or mystical state. We then look what energy states are explored during meditation: is it the "hyperfine" structure of its base state? is there a love ecstatic state? a very high energy structure mystical state? On one hand we illustrate these hypothesis from the experience of a large part of mystical traditions such as Hinduism or Buddhism and on another hand from contemporary cognitive sciences. In addition, quantum mechanics indicates that any interaction between energy levels is mediated by a boson of exchange. So we aim to identify the nature of this boson linking the various human being energy levels.
\end{abstract}

Mots clés : hypothèse fractaquantique.

1 Titulaire d'un master en physique atomique et statistique, professeur des universités (mathématiques) au Conservatoire National des Arts et Métiers à Paris, membre d'Association Française de Science des Systèmes Cybernétiques, Cognitifs Et Techniques ; courriel francois.dubois@cnam.fr.

2 Docteur en philosophie comparative, instructeur MBSR (Mindfulness-Based Stress Reduction), membre de l'Association pour le Développement de la Mindfulness (ADM) et du Center For Mindfulness in Medicine, Health Care, and Society (CFM) ; courriel k.miquel@free.fr.

3 Première version 9 juillet 2014. Contribution présentée au congrès international de systémique à Valencia le 16 octobre 2014. Edition française corrigée pour publication dans le journal Acta Europeana Systemica. 


\section{Etat fondamental et hypothèse fractaquantique}

Ce travail se place dans le cadre de l'hypothèse fractaquantique ${ }^{4}$ : l'approche quantique est applicable à toutes les échelles indivisibles de la nature, quelle que soit leur taille. De plus, la question sous-jacente est celle d'un modèle quantique pour l'Homme, sachant qu'un modèle quantique ne s'intéresse pas aux individus particuliers, mais au contraire à ce qu'ils ont en commun, et les rend par cette approche « indiscernables ».

Nous sommes guidés par l'analogie atomique. On sait qu'un atome est, du point de vue quantique, bien décrit par ses niveaux d'énergie. Ceux-ci peuvent être calculés comme les valeurs propres de l'opérateur Hamiltonien qui décrit l'interaction entre l'atome et l'environnement électromagnétique extérieur. Ainsi, l'atome dispose de niveaux d'énergie proposés par Niels Bohr (1913) et parfaitement calculables (au moins pour l'atome d'hydrogène) grâce à la mécanique quantique ; un niveau fondamental $|0\rangle$, un premier niveau excité $\mid 1>$, un second noté $\mid 2>$, etc. Quand l'atome passe du niveau zéro au niveau un, il absorbe l'écart d'énergie correspondant, absorbant une onde de fréquence $v$ selon la relation

$$
\mathrm{h} v=\varepsilon_{1}-\varepsilon_{0},
$$

où h est la constante de Max Planck. Quand l'atome passe du niveau $\mid 1>$ au niveau fondamental $|0\rangle$, il réémet une onde de fréquence selon la relation (1).

L'analogie première rappelée ici s'intéresse aux états excités de l'Homme, dans cette description quantique. Nous admettons que la vie quotidienne, avec ses joies et ses peines, ses désirs et ses frustrations, correspond à autant de modulations d'un état fondamental. Cet état fondamental pourrait se définir dans une première approche, en creux, par rapport à tous les états de plus grande excitation : ce sont les «états courants » de la vie profane ou quotidienne, faits d'excitations faibles, instables, discontinus, changeant selon les échanges d'énergie effectués à chaque moment avec le monde extérieur, sans que le sujet ordinaire en ait forcément conscience. On peut les rapprocher de ce que Christophe André ${ }^{5}$ nomme des " états d'âmes ", ces mélanges de sensations, pensées et émotions qui correspondent à chaque moment à un état d'énergie temporaire de ma relation au monde, changeant à l'occasion de chaque nouvelle configuration, en étant alors remplacés par un autre état, avec à chaque fois des fluctuations et des micro-sauts d'énergie correspondants. Nous appellerons cet état fondamental d'excitation faible et désordonnée de la vie courante, l'« état quotidien agité», noté " état $\mid 0>$ », et y reviendrons par la suite.

Cette structure de base étant posée pour un atome (on parle techniquement des orbitales moléculaires), la physique nous apprend qu'une « structure fine ${ }^{6}$ existe pour chaque niveau. L'écart d'énergie entre les sous-niveaux qui composent cette structure fine est beaucoup plus faible que la différence $\varepsilon_{1}-\varepsilon_{0}$ de la relation (1). Toujours avec la même analogie, nous proposons ici qu'une succession d'états d'énergie $\varepsilon_{\mathrm{j}}$ compose en fait le niveau fondamental $\mid 0>$ de l'Homme. C'est comme pour l'atome, une « structure fine », même si nous n'avons pas à ce jour de représentation explicite à proposer. Mais ainsi qu'évoqué plus haut, les divers sentiments, la colère, la tristesse, les désirs, etc. sont autant de " sousétats excités » de l'état fondamental. On sait qu'une colère demande de l'énergie, puis retombe. Même chose pour les désirs et leur assouvissement, la tristesse ou la peur.

Un vrai chantier de spectroscopiste s'ouvre ici de classer par écart d'énergie ces différents états. A la suite de Descartes, Spinoza a été le premier à emprunter cette voie, puisque toute son éthique consiste à montrer comment l'éthique invite à s'écarter des passions tristes empreintes de ressentiment, pour privilégier la Joie qui correspond au maximum d'intensité d'être ${ }^{7}$. La roue des émotions de Plutchik

4 François Dubois, « Hypothèse fractaquantique », Res-Systemica, volume 2, 2002.

5 Christophe André, Les états d'âme, pour un apprentissage de la sérénité, Odile Jacob, Paris, 2009.

6 Voir par exemple l'ouvrage Mécanique quantique de Claude Cohen-Tannoudji, Bernard Diu et Franck Laloë, Hermann, Paris, 1977.

7 Voir Antonio Damasio : Spinoza avait raison : joie et tristesse, le cerveau des émotions, Odile Jacob, Paris, 2003. Descartes avait ouvert la voie avec son Traité des passions, où il recensait six passions de base : 
permet quant à elle de montrer comment les huit émotions de base (joie, peur, dégoût, colère, tristesse, surprise, confiance, anticipation) peuvent chacune passer par trois degrés ou variations d'intensité différentes ${ }^{8}$. De la contrariété, à la colère puis à la rage, tout comme de l'appréhension, à la peur puis à la terreur, ou en positif de la sérénité, à la joie puis à l'extase, il y a à chaque fois une fluctuation accompagnée probablement de micro-sauts d'énergie correspondant.

L'étude finlandaise de Lauri Nummenmaa ${ }^{9}$, de la faculté des sciences d'Aalto, publiée dans les Comptes rendus de l'Académie des sciences américaine a récemment permis d'approfondir et nuancer ce classement des émotions, en montrant à la manière de Spinoza que selon leur nature, elles affectent plus ou moins subjectivement et énergétiquement le corps. Plus de sept cents volontaires de plusieurs pays ont été invités à montrer sur une silhouette humaine les parties de leur corps qui se trouvaient suractivées, ou au contraire diminuées, lorsqu'ils ressentaient l'une de sept émotions, dont les cinq émotions actuellement reconnues par les chercheurs comme primaires : joie, tristesse, colère, peur, dégoût. Le résultat montre que l'activation du corps va de " très faible " à " faible " lorsqu'on passe de la dépression à la tristesse, au mépris ou à la honte, avec une sous-activation à chaque fois des membres inférieurs et une légère activation du haut du corps. A l'inverse, l'activation corporelle est de plus en plus forte, essentiellement dans les zones supérieures du corps, lorsqu'on passe du dégoût, à la peur et à la colère. Les deux émotions du bonheur et surtout de l'amour correspondent aux activations les plus intenses, cette fois-ci de l'ensemble du corps.

Observons que l'énergie, les écarts d'énergie des niveaux atomiques, et par extension des niveaux d'excitation de l'Homme, sont de même nature que l'énergie mécanique d'un moteur. Il s'agit d'un concept physique bien compris depuis les travaux pionniers de Sadi Carnot ${ }^{10}$ et Josiah Gibbs ${ }^{11}$ au dixneuvième siècle.

\section{De l'état fondamental vers les états amoureux et mystiques}

Si nous nous nous intéressons maintenant plus particulièrement aux états d'excitation qui font sortir de cet état fondamental, nous faisons l'hypothèse que le premier état excité $\mid 1>$ est l'état amoureux. De façon analogue à la relation (1), il "donne de l'énergie », place la personne dans un état très particulier. Nous l'avons tous éprouvé et savons qu'après un certain temps, le temps de la passion, l'être « retombe » de l'état amoureux à l'état ordinaire. Il a été décrit par toutes les cultures depuis la nuit des temps. En écrivant ces lignes à Trouville, nous pensons aux livres de Christian Bobin ${ }^{12}$, à Alain de Botton, écrivain anglais d'origine suisse qui développe une Petite philosophie de l'amour ${ }^{13}$, à Pierre de Ronsart : " Mignonne allons voir si la rose... » ${ }^{14}$. Pour la déception amoureuse, le fait de rester dans cet état $\mid 1>$ alors que l'autre n'adhère plus à la relation, nous renvoyons aux œuvres des poètes, aux chanteurs populaires et par exemple à « l'écharpe » ${ }^{15}$ de Maurice Fanon, ou à « mon amant de Saint Jean ${ }^{16}$.

admiration, haine, amour, désir, joie, tristesse.

8 Robert Plutchik et Hope R. Conte, Circumplex Models of Personality and Emotions, Herausgeber, 1997.

9 Lauri Nummenmaa, Enrico Glerean, Riitta Hari and Jari K. Hietanen. Bodily maps of emotions, Proceedings of the National Acadeny of Science, vol. 111, no. 2, p. 646-651, doi: 10.1073/pnas.1321664111, New York, 2013.

10 Sadi Carnot. Réflexions sur la puissance motrice du feu et sur les machines propres à développer cette puissance, Bachelier, Paris, 1824.

11 Josiah Gibbs. Elementary principles in statistical mechanics,? Charles Scribner's sons, New York, 1902.

12 La part manquante, Gallimard, 1989 ou Le Très-Bas, Gallimard, 1992, pour citer deux fondamentaux...

13 traduit de l'anglais par Raymond Las Vergnas, Presses Pocket, Paris, 1997.

14 Ode XVII à Cassandre, Les Quatre premiers livres des Odes de P. de Ronsard Vandomois, Dediés au Roy, A Paris. Chez la veufve Maurice de la Porte, au clos Bruneau, à l'enseigne sainct Claude. 1555. Avec privilege du Roy. (Achevé d'imprimer le xxv. de Janvier 1555.).

15 Avec Fanon, CBS, 1963.

16 Paroles de Léon Angelliaume (dit Léon Agel) et musique d'Emile Carrara, déposée à la SACEM le 9 avril 1940 sous le titre « Les barbeaux de Saint-Jean », enregistrée par Jane Chacun en juin 1942 chez Odéon sous 
Notre idée est de ne pas arrêter la description quantique aux deux états décrits plus haut, l'état fondamental et l'état amoureux. Platon, dans le Banquet, n’invite-t-il pas à voir le désir, puis Eros, l'état amoureux, comme un tremplin vers la quête du Beau, du Bien et du Vrai qui correspondent à des états supérieurs de contemplation, auxquels l'amoureux participe sans même le savoir ? Nous proposons que l'état mystique soit considéré comme " second état excité » de l'Homme et nous le notons |2>. L'expérience de l'état mystique est plus rare que celle de l'état amoureux. Elle est décrite de façon fort poétique par Thérèse d'Avila («Le château de l'âme » ${ }^{17}$ ) ou Jean de la Croix ( « La nuit obscure $"{ }^{18}$ ) dans la culture chrétienne, chez les poètes soufis, ou de façon plus proche de nous par Karlfried Graf Dürkheim («L'esprit guide ${ }^{19}$ ) ou Jiddu Krishnamurti ${ }^{20}$. Nous pensons que l'état mystique est aussi naturel que l'état amoureux. Etat plus « excité » d'emblée: les mystiques parlent souvent de l'impression d'être "ravis ", transportés hors d'eux, avec parfois des tremblements du corps, ils peuvent rentrer dans des phénomènes proches de la transe, tant l'excitation est forte. Ces expériences sont la plupart du temps fugaces et fragiles car ces états sont difficilement compatibles avec l'agitation et la violence du quotidien. A priori, cet état est limité dans le temps et Jean de la Croix parle de « nuit obscure » pour décrire ce choc du retour à l'état « fondamental ».

Il est probablement envisageable d'aller au-delà de l'état mystique, au delà du niveau $\mid 2>$, vers un état $\mid 3>\ldots$ Que devient, dans ce stade, l'état de sur-excitation dont nous parlions ? Se dilue-t-il en retournant à un état fondamental apaisé qui n'est plus traversé par aucune micro-excitation, ou en s'immergeant dans un état d'énergie supérieure ? S'agit-il, simplement, d'une modulation de l'expérience d'excitation, comme une structure non plus fine, mais « hyperfine », comme en physique quantique?

Les atomes ont en effet, en plus de leur structure fine, une structure hyperfine liée au couplage du moment magnétique du proton avec le moment orbital de l'électron et à d'autres effets quantiques ${ }^{21}$. Ce sont là encore des transitions bien connues et largement étudiées par les physiciens atomistes. Fautil parler d'une structure hyperfine de l'excitation mystique, ou d'un nouveau saut comparable aux autres sauts quantique, vers un état d'excitation apaisé ou fusionné dans un stade énergétique encore supérieur, de niveau $\mid 3>$ ?

Pour avancer dans ce questionnement, nous proposons de nous intéresser non plus aux expériences extatiques des mystiques, mais aux expériences de méditation qui ont l'avantage d'avoir cartographié, depuis maintenant plus de deux millénaires, les multiples paysages mentaux que peut traverser un esprit qui s'entraîne régulièrement. D’autant que, depuis l'essor récent des neurosciences, la méditation est elle-même devenue l'objet de nombreuses études scientifiques.

\section{Les différente étapes et expériences de méditation}

En quoi consiste la méditation ? Selon Francisco Varela ${ }^{22}$, qui a dégagé les structures communes aux méditations bouddhistes, soufi et aux prières orthodoxes, il s'agit toujours du même mouvement de mobilisation et de refocalisation de l'attention, qui se fait en trois temps : il s'agit d'abord de

le titre « Mon costaud de Saint-Jean » puis par Lucienne Delyle le 7 juillet 1942 chez Columbia sous le titre « Mon amant de Saint-Jean ».

17 Las Moradas o il Castillo interior, Teresa de Ávila, 1577. Le Château intérieur, ou les Demeures de l'âme, traduction André Félibien (1619-1695), Paris, 1670.

18 La noche oscura, Juan de Yepes Álvarez, 1584.

19 Entretiens avec Franz Woerly, Albin Michel, Paris, 1984.

20 Voir par exemple The First and Last Freedom, Foreword by Aldous Huxley, Gollancz, London, 1954, ou Krishnamurti's Notebook, Gollancz, London, Harper \& Row, New York, 1976.

21 comme le couplage avec le spin de l'électron et au terme de contact entre l'électron et la « frontière » du proton, voir Cohen-Tannoudji et al., op cit.

22 Voir par exemple Natalie Depraz, Francisco Varela et Pierre Vermersch : A L'epreuve De L'experience: Pour Une Pratique Phénoménologique, Zeta Books, 2011. 
suspendre la focalisation de son attention vers le monde regardé comme un objet externe qui vient nous solliciter perpétuellement, ce qui amène à se concentrer (samatha dans le bouddhisme) de manière calme, en revenant à soi, notamment à son souffle. Cette suspension permet dans un deuxième temps d'inverser le regard et le flux de l'attention, de re-diriger cette dernière vers ses sensations internes, vers toutes les micro-excitations corporelles ou mentales qui se déroulent à chaque instant, en observant (vipassana) selon Bitbol ${ }^{23}$ leurs variations aussi infimes qu'impermanentes, sans se laisser emporter par elles. A force de maintenir l'attention re-dirigée vers soi, se crée et se creuse un nouvel état qui marque une véritable rupture de niveau de l'état d'excitation habituel. Il se caractérise par le troisième moment inhérent selon Varela et Bitbol à toute pratique de méditation : l'attention devient juste ouverte et disponible, en simple «état d'accueil » écrivent-il. C'est, selon eux et selon les expériences des personnes s'adonnant à la méditation, un état d'attente ouverte, sans objet ou connaissance du contenu qui va se révéler, indépendamment de toute trace d'excitation. Ce temps de vide relatif peut être très bref, durer quelques secondes ou centièmes de secondes, plusieurs minutes, sinon plus, le temps que de nouveaux objets ou stimuli viennent inéluctablement se présenter dans le champ de la conscience. Selon Bitbol qui reprend les termes de Romano ${ }^{24}$, le plus déroutant et parfois angoissant pour les débutants en méditation, c'est d'aborder cette terra incognita des événements intérieurs qui vont se produire sans savoir à l'avance ce qui va se passer, d'accepter ce bref temps vide qui va les laisser émerger, de se mettre « en immersion dans l'apparaître », plutôt qu'en " prise de distance réflexive » par rapport à celui-ci ${ }^{25}$.

Que se passe-t-il maintenant, pendant la méditation ? Tout... et rien! Des pensées, des émotions, des sensations agréables ou douloureuses surgissent, l'endormissement ou l'agitation mentale guettent. Le principal est d'apprendre à ne pas se laisser emporter par eux, en découvrant progressivement qu'il y a plusieurs stades possibles dans la méditation, avec à chaque fois des corrélations intéressantes au niveau du cerveau.

On peut voir ces pensées comme des «bosons virtuels », qui forment un concept classique pour les calculs de théorie quantique des champs. S'il y a communication, échange entre deux partenaires ou deux entités, un boson d'interaction est transmis et reçu ${ }^{26}$. Dans le cas d'une non-communication, comme une pensée du sujet sans communication explicite, le boson est émis et ré-absorbé par la même entité, ce qui est caractéristique des bosons virtuels ${ }^{27}$.

\section{Premier type d'expérience méditative possible, et exploration de l'état fondamental}

Dans le premier stade, la méditation ouvre à la découverte des micro-sensations internes auxquelles on ne fait pas habituellement attention. Immobile, coupé des sollicitations du monde extérieur, l'attention descend en effet du niveau de la conscience ordinaire soumise à des successions d'états d'âme et d'excitation multiples sans en avoir forcément conscience, à un état de présence attentive à ce qui se passe au sein de soi. Non pas en adoptant la posture externe de l'observateur qui décomposerait analytiquement ses sensations internes comme autant d'objets à observer, mais en se servant de sa capacité de présence intuitive à soi, pour revenir en deçà de la coupure sujet-objet comme l'écrit Michel Bitbol ${ }^{28}$. On apprend à voir passer dans le champ de sa conscience, sans se laisser emporter par eux, des micro-sensations physiques internes, des émotions et pulsions partielles, des pensées qui surgissent et disparaissent de manière aussi fugace que les bosons, sans laisser de trace.

23 Michel Bitbol : La conscience a-t-elle une origine ?, Flammarion, 2014.

24 Claude Romano, Le chant de la vie : phénoménologie de Faulkner, Gallimard, Paris, 2005.

25 Michel Bitbol, La conscience a-t-elle une origine ? Flammarion, 2014, p 168.

26 Voir par exemple le livre de Claude Itzykson et Jean-Bernard Zuber, Quantum Field Theory, McGraw Hill, 1980.

27 Nous renvoyons aussi à la contribution « Pistes fractaquantiques » de l'un d'entre nous, Res-Systemica, volume 4, numéro 2, décembre 2004.

28 Michel Bitbol, De l'intérieur du monde, pour une philosophie et une science des relations, Flammarion, 2010 
Cette capacité à observer attentivement le surgissement, le déploiement et la disparition des multiples émotions ou sensations qui traversent notre organisme à chaque instant, sans se laisser entraîner par eux, correspond à une modification du fonctionnement habituel du cerveau. Les études neuroscientifiques actuelles montrent en effet que pendant la méditation Mindfulness dite de pleine conscience, les zones de la pensée rationnelle (aire de Broca et de Wernicke) sont en moindre activité, alors que les zones des perceptions internes de notre propre corps sont au contraire stimulées et en suractivité : le cortex fronto-limbique et le cortex fronto-pariétal qui favorise l'introception ou perception de ses sensations internes, le cortex cingulaire antérieur qui permet de renforcer les sensations corporelles et les réponses à la douleur ${ }^{29}$, le cortex préfrontal gauche qui favorise la gestion du stress et les émotions de bien-être, notamment en réponse à des émotions négatives comme la tristesse. Plus on médite et devient « expert ", plus des modifications se produisent dans le cerveau, qui se remodèle, avec, entre autres, une diminution de l'amygdale qui est le siège des réactions automatiques en cas de stress, mais aussi un épaississement notable de la substance grise permettant un meilleur contrôle du cortex, un allongement des télomères responsables du maintien de la vie cellulaire (le stress raccourcissant au contraire ces télomères et la durée de vie correspondante des cellules), et enfin une meilleure capacité pour fixer durablement son attention.

Lorsque le méditant parvient à installer une certaine stabilité de l'esprit, nous faisons l'hypothèse que cette observation méditative très fine de ses états énergétiques internes correspond, selon le modèle de la physique quantique, à une descente de l'état quotidien agité, à ce que nous nommerons un « état tranquille », dans lequel il est possible de devenir conscient des structures fines, voire hyperfines de l'état fondamental de l'homme et des micro-sensations et excitations qui se succèdent perpétuellement en lui. Avec le même constat, en physique quantique et dans les courants de méditation bouddhiste : il n’y a pas de flux continu, mais des apparitions discrètes et éphémères, suivies de disparitions et nouvelles émergences énergétiques, à chaque fois discontinues.

Passer de l'état agité à l'exploration des micro-mouvements et structures fines, voire hyperfines, de l'état fondamental, implique une gymnastique de l'attention, qui, progressivement au fil des méditations, inculque un nouveau cycle et mécanisme cognitif en quatre temps qui selon Antoine Lutz force différentes zones du cerveau à travailler ensemble, en calmant et fixant progressivement l'attention ${ }^{30}$. Au vagabondage mental qui se produit habituellement par un fonctionnement du cerveau " par défaut ", en mode automatique au niveau du cortex sensoriel et moteur, succède pendant la méditation une prise de conscience de ce vagabondage mental au niveau du cortex cingulaire, du cortex somatosensoriel et de l'insula antérieur, ce qui permet d'arrêter un moment le flux mental qui nous emporte toujours ailleurs. Cette prise de conscience permet à l'attention de se détacher de l'objet du vagabondage, en mobilisant cette fois le cortex préfrontal dorsolatéral et les régions pariétales antérieures. Elle peut alors se refocaliser de façon soutenue et calme au niveau du cortex préfrontal, avec l'établissement d'une présence attentive large et ouverte à ce qui se passe.

\section{Deuxième type d'expérience méditative possible, et parallèle avec l'état amoureux}

Si l'apprentissage de la méditation correspond ainsi à un approfondissement plus fin de l'état fondamental de l'homme, certains types de méditation permettent, dans de nombreuses traditions méditatives, d'explorer de nouveaux états plus excités, qui en appellent probablement aux états amoureux préalablement présentés, en offrant toutes les caractéristiques du stade $\mid 1>$ amoureux, voire

29 Voir le résumé des différentes études en neurosciences concernant les effets de la méditation sur le cerveau, dont celle de Katia Rubia, Université de Londres ; in Christophe André : « La méditation de pleine conscience », in Cerveau et psycho n²41, 2010.

30 Voir l'étude par IRM de Wendy Hasenkamp, Université d'Atlanta : Mind wandering and attention during focused meditation : a fine-grained temporal analysis of fluctuating cognitive states, in Neuroimages, vol 59 (1), 2012. Etude commentée par Antoine Lutz in Cerveau et psycho n52, août 2012. 
du stade $\mid 2>$ mystique. Pour étayer notre hypothèse, nous prendrons des exemples puisant dans les deux principales traditions orientales qui s'opposent pourtant souvent : l'hindouisme et le bouddhisme.

Dans l'hindouisme, la bhakti yoga meditation, ou voie de la dévotion, est célèbre depuis qu'elle a été thématisée pour la première fois dans la Bhagavad Gita ${ }^{31}$, entre le $5^{\circ}$ et le $1^{\circ}$ siècle avant J.C. Essentiellement tournée vers Vishnou ou vers Krishna, cette bhakti permet de développer et cultiver, dans sa vie quotidienne comme dans sa méditation, un état de transport amoureux avec sa divinité de cœur. La bhakti implique en effet un complet abandon à sa divinité, en installant un rapport de personne à personne avec son Dieu, le dévot recevant ou découvrant une intensité d'énergie et d'amour en rupture avec les sentiments habituels du monde profane.

Comme on s'en doute, dans les stades les plus avancés, une telle dévotion amoureuse se prolonge naturellement en état mystique, puisqu'il s'agit alors de s'unir et se fondre dans l'amour infini de son Dieu, les dévots de Vishnou ou Krishna se remarquant parfois dans les rues par les véritables transports amoureux et énergétiques qui les saisissent pendant qu'ils psalmodient et méditent, cherchant à faire perdurer cet état d'extase amoureuse, avant que cet état ne retombe fatalement, comme cette Nuit obscure dont parle St Jean de la Croix. Dans les cas pathologiques, notamment dans la bhakti bengali comme le remarquait Esnoul ${ }^{32}$, ces états de ferveur seconds peuvent dépasser les cadres de la méditation pour déborder en phénomène de foule de dévots en liesse, avec des troubles nerveux ou fonctionnels provoqués par des excitations émotionnelles extrêmes.

Dans le bouddhisme, les méditations de la compassion et de l'amour bienveillant mises en avant par Matthieu Ricard ${ }^{33}$ pour le bouddhisme tibétain, consistent à développer un sentiment d'amour infini, sans limite, en étendant le sentiment de bonté, d'empathie et d'amour compassionnel, même envers des gens qu'on n'aime ou ne respecte pas. Pendant de telles méditations cultivant la compassion, le méditant passe par des sensations fortes et parfois désagréables qui le font passer dans des états psychiques d'intensité et d'énergie supérieures, avec des activations fortes de certaines zones du cerveau qui ont pu être mesurées par les neurociences. Pendant la méditation de l'amour bienveillant, Richard Davidson et Antoine Lutz ${ }^{34}$, du CERN, ont ainsi enregistré une activation supérieure des régions du cerveau gérant l'empathie face à la souffrance d'autrui (l'insula antérieure et le cortex cingulaire antérieur, mais aussi les trois zones qui nous permettent de nous mettre à la place d'autrui: cortex préfrontal médian, sillon temporal supérieur, jonction temporo-pariétale).

Les méditants expérimentés ressentent encore plus cette compassion et cette souffrance d'autrui, avec une activation supplémentaire du cortex somatosensoriel qui les fait s'inscrire dans leur corps. Selon Matthieu Ricard, d'autres études ${ }^{35}$ pendant cette méditation de l'amour bienveillant témoignent logiquement de la diminution d'activité de l'amygdale qui gère l'agressivité et la peur, alors que les zones liées à l'empathie comme l'insula sont plus actives, augmentent de taille, en créant plus de connexions neuronales et remodelant ainsi le cerveau. Le sentiment d'amour bienveillant va encore plus loin que la simple augmentation de l'empathie, selon les études relatées par Matthieu Ricard, notamment en favorisant et développant l'ocytocine, cette peptide de l'amour maternel fabriquée par l'hypothalamus.

31 Bhagavad Gita, traduction/commentaires d’A.M. Esnoul et O. Lacombe, Fayard, 1972.

32 A.M. Esnoul : Le courant affectif à l'intérieur du courant brahmanique ancien, Bulletin de l'Ecole française de l'extrême Orient, 1956, p. 152.

33 Matthieu Ricard, Plaidoyer pour l'altruisme, la force de la bienveillance, éd Nil, 2014

34 Voir Richard Davidson et Antoine Lutz, étude par IRM de 2008, résumée par Antoine Lutz in : Le cerveau méditatif, Cerveau et psycho n52, août 2012.

35 Matthieu Ricard, Plaidoyer pour l'altruisme, la force de la bienveillance, éd Nil, 2014. 


\section{Troisième type d'expérience méditative possible, approches de l'état mystique}

Si ces méditations basées sur l'amour semblent bien permettre de passer de l'état fondamental à l'état amoureux de niveau $\mid 1>$, voire à l'état mystique $|2\rangle$, existe-t-il des pratiques méditatives non dévotionnelles, qui feraient plus spécifiquement passer au niveau $\mid 2>$ dit mystique ? Il nous semble que c'est le cas des états avancés de concentration dhyana et de contemplation samadhi, qui ont été catalogués par toutes les traditions de méditation des deux grands courants hindouistes et bouddhistes. Depuis plus de 2000 ans, ces états de conscience ont en effet été largement répertoriés ${ }^{36}$ et présentés, tantôt dans l'hindouisme comme l'accession à de nouvelles strates d'énergie et de conscience supérieure, tantôt pour le bouddhisme comme des stades de conscience et d'obtention de pouvoirs supérieurs dont il faut se méfier et auxquels il ne faut pas s'arrêter.

Présentons les premiers états de conscience avancée qu'on retrouve dans ces diverses traditions, en nous arrêtant pour la commodité sur les termes et stades décrits par le bouddhisme ${ }^{37}$, et plus spécifiquement dans les dhyanas ou jhanas, états d'absorption plus ou moins ultimes auxquels on peut parvenir après de longues médiations et concentrations samadhi. Le premier état dhyana qui nous semble être la définition même de l'état mystique donnée au début de cet article est l'expérimentation d'abord d'un état de pure énergie intérieure ou excitation virya, en sentant surgir au plus profond de soi un jaillissement interne d'énergie. Ce jaillissement d'excitation et d'énergie peut être vécu avec des signes faibles comme de la chaleur, des picotements, la chair de poule, ou provoquer comme chez les mystiques des mouvements plus violents qui emportent le méditant littéralement, quasiment hors de lui, donnant l'impression d'être transporté, jusqu'à quasiment pouvoir léviter ou avoir l'impression de sortir de son corps, dans un état de ravissement et de joie pure priti ou passadhi, qui témoigne alors de l'accès au second état.

Cet état de joie peut se prolonger quasiment naturellement en un troisième état de bonheur pur sans sensation ou excitation corporelle, avec à nouveau une rupture de niveau mental d'expérience, et l'accès à un quatrième état prasrabdhbi d'équanimité, de sérénité et de paix immuable. Lequel peut amener à se laisser absorber totalement dans de nouveaux états dhyana ou jhana plus subtils encore selon les textes : en cinquième stade, la dilution ou absorption dans un espace illimité ; en sixième, l'absorption dans un état de conscience illimitée ; en sept et huit, l'absorption dans d'autres espaces vides de tout être ou de toute forme, parfaitement silencieux, au-delà des perceptions et des non perceptions.

Les textes hindous et les différentes étapes du yoga présenteront des variantes et inversions des termes, en distinguant par exemple selon Mircea Eliade ${ }^{38}$, après la concentration dhyana, des samadhi de plus en plus raffinés: d'abord, dans le samadhi avec support, une enstase ou affranchissement des limites habituelles, qui se fait en conservant encore le support de l'objet sur lequel on médite. Cela amène ensuite à obtenir des pouvoirs yogiques miraculeux ${ }^{39}$, et enfin lorsqu'on les dépasse, au samadhi ultime sans aucun support, qui correspond à une illumination parfaite et soudaine, une enstase au plus profond de soi, dans un état de conscience supérieur, « saturé par une intuition totale et directe de l'être ${ }^{40}$ "

Il n'est pas étonnant que ces états d'absorption atteints par la méditation retrouvent les états dont témoignent les mystiques. Dans son parcours spirituel, Sainte Thérèse atteint ainsi dans la cinquième

36 Pour l'hindouisme : les yoga sûtra, entre -200 et +500 , ont codifié et compilé pendant probablement plusieurs siècles les expériences et stades développés par les yogins pendant leurs exercices de yoga et de méditation. Pour le bouddhisme : l'Abidharma, entre le $1^{\circ}$ et le $5^{\circ}$ siècle, constitue de même un recueil impressionnant de textes et de commentaires sur les expériences pyschologiques et philosophiques expérimentées par le Bouddha et par les méditants bouddhistes. Michel Bitbol, dans ses recherches sur la conscience, s’appuie entre autres sur ce corpus.

37 De multiples descriptions de ces différents états existent. Nous renvoyons, pour plus de commodité, à leur description par J Kornfield : Bouddha mode d'emploi, Belfond, 2011, p. 442 et suivantes.

38 Mircea Eliade : Le yoga, immortalité et liberté, Payot, 1954, p. 107.

39 Patanjali, -200 : Yoga-Sutra, III, 16, sq. 
demeure de son âme une attention pure qui succède à une félicité infinie, ce qui correspond selon Bitbol ${ }^{41}$ au troisième dhyana bouddhiste du retrait de la conscience des sensations corporelles pour devenir attention pure, juste après un sentiment de joie et de félicité. Pour faire éclore de tels états, la capacité de fixer son attention, puis de se laisser aller à l'abandon, est capital comme le remarquait également la mystique Jeanne Guyon au $13^{\circ}$ siècle : " L'abandon est la clef de tout l'intérieur ${ }^{42}$ "

Peu importe la différence des stades et des termes dans le yoga ou dans le bouddhisme, qu'on privilégie le samadhi ou le dhyana. Le principal est de prendre conscience que ces deux traditions ont cartographié depuis longtemps des états de conscience qui retrouvent les états mystiques de niveau " deux » de nos hypothèses quantiques, avec à chaque fois le même changement de degré, une rupture de niveau, un élargissement et une intensification énergétique de la conscience, tant dans des aspects physiologiques que cognitifs.

Des études menées en neurosciences permettent, là encore, d'approcher indirectement et comparativement les transformations énergétiques qui se passent probablement dans le cerveau, lorsqu'il atteint en méditation de tels états, pendant une durée plus ou moins brève. Les mesures menées sur des méditants entrainés ${ }^{43}$ montrent en effet à la fois l'accroissement des ondes alpha et thêta de la relaxation profonde et du sommeil paradoxal, mais aussi l'augmentation des ondes gamma reflétant l'activation et la mobilisation des ressources neuronales permettant l'effort mental et l'émergence d'un éclair intuitif global. Tout comme elles permettent, selon Chaskalson, d'accumuler quotidiennement des données sonores, visuelles et cognitives, pour brusquement comprendre par exemple en un éclair : c'est un train, ces mêmes ondes provoquent ces éclairs de compréhension intuitive que connaissent les grands savants, artistes ou méditants. C'est le " aha! », le « eureka » qu'on retrouve qui durent quelques millièmes de secondes, qui permettent de voir brusquement la réalité autrement. Selon Chaskalson, sans même parler des états de samadhi ou de dhyana que nous avons présenté, l'entrainement mental de la méditation permet aux moines d'avoir le même type d'illumination et d'éclair intuitif, avec une vision large et globale qui peut durer jusqu'à cinq minutes.

Comment l'expliquer, ou en rendre compte en neurosciences ? Les études menées par Lutz ${ }^{44}$ dans son laboratoire de recherche l'Université du Wisconsin, sur des méditants experts ayant à leur actif plus de 10.000 heures de méditation, montrent que les zones du stress localisées dans l'amygdale sont beaucoup moins mobilisées, même en cas de stress objectif (sons agressifs envoyés), ce qui leur permet d'obtenir une stabilité émotionnelle et attentionnelle. Lors d'autres études sur des méditants encore plus chevronnés, totalisant plus de 50.000 heures de méditation, Lutz ${ }^{45}$ montre également que la synchronisation des ondes gamma est effectivement beaucoup plus nette que chez des individus lambda, ce qui témoigne de niveaux de conscience "augmentés », avec plus d'intégration des différentes zones cérébrales qui se mettent à fonctionner en synergie, de manière plus fluide et plus ouverte...

40 Mircea Eliade : Histoire des croyances et des idées religieuses, Payot, 1978, tome 2, p. 70. Voir aussi la description par Zimmer de ces mêmes états de samadhi selon le grand philosophe vedanta Cankara, au $8^{\circ}$ siècle : Zimmer : les philosophies de l’inde, Payot, p. 342.

41 Michel Bitbol : La conscience a-t-elle une origine ? Flammarion, 2014, p. 175.

42 Madame Guyon : Le moyen court et autres récits spirituels, textes édités par M.-L. Gondal, Grenoble, 1685, Jérôme Millon, Coll. « Atopia », 1995, cité par Bitbol, infra.

43 Michel Chaskalson: Méditer au travail pour concilier sérénité et efficacité, les Arènes Eds, 2013, p 113.

44 Antoine Lutz : Long term meditators self-induce high amplitude gamma synchrony during mental practice, Proc Nat Acad Sciences, Usa, vol 101, 46, 2004.

45 Antoine Lutz : Le cerveau méditatif, Cerveau et psycho n52, juillet-août 2012. 


\section{Les apories des états mystiques: troisième état |3>, ou retour à l'état fondamental ?}

Si l'existence d'états mystiques correspondant à un « saut » dans un niveau d'énergie supérieur semble ainsi démontré, que deviennent ces états mystiques : peuvent ils se dépasser en un stade ultérieur que nous nommerions un état quantique de niveau $\mid 3>$, ou retournent-ils finalement à l'état fondamental ?

L'hindouisme et le yoga semblent militer en faveur de la possibilité d'atteindre un état ultime de niveau $\mid 3>$. Dans l'hindouisme, l'état mystique pointe naturellement vers la contemplation, vers des sentiments d'union ou de fusion dans une énergie supérieure, voire divine, qui va au-delà des états d'absorption précédemment décrits. C'est ce qu'indiquaient déjà les textes hindouistes les plus anciens, Bitbol rappelant ainsi que la Chandogya Upanishad affirmait déjà six siècles avant J.C. que "la méditation dhyana est plus que la conscience ${ }^{46}$. Pour les hindous, l'expérience ultime est en effet celle de la fusion et de la résorption dans l'océan primordial, pensé sur le modèle de la vague ou de la goutte d'eau (atman ou principe ultime en soi), qui retourne se fondre dans l'océan primordial de l'absolu (Brahman). Cankara, grand philosophe non dualiste qui appartient à la tradition hindouiste du Vedanta au $8^{\circ}$ siècle après J.C., s'inscrira dans la même lignée lorsqu'il distinguera selon son commentateur Zimmer les différents samadhi avec, puis sans support, pour finir finalement par une fusion océanique dans l'absolu : «Dans le samadhi de premier type savikalpa, avec pleine conscience de la dualité sujet-objet, la vitalité oscillante de la conscience assume la forme de Brahman, mais demeure consciente d'elle-même, jouit d'une extase bénéfique... Samadhi nirvikalpa, absorption sans conscience, c'est l'immersion dans le Soi, sans distinction sujet objet, comme les vagues s'évanouissent dans l'eau ${ }^{47}$ ".

Mais cette fusion dans l'infini qui semble de niveau quantique $\mid 2>$, voire $\mid 3>$, n'est-elle pas en même temps une résorption dans un état fondamental, naturellement apaisé et informel, qui évoque à nouveau le paradoxe d'un niveau $\mid 0>$ ? C'est ce que semble indiquer le bouddhisme pour lequel les différentes étapes de résorption dhyana ne constituent pas une fin en soi : elles permettent simplement d'abandonner le jeu des catégories et des projections mentales habituelles, de se soustraire aux excitations qui continuent de se produire sans qu’on en soit désormais affecté ou obligé de réagir, en se contentant de revenir à un état originellement apaisé, où on laisse juste le spectacle du monde apparaître ici et maintenant, dans un perpétuel présent. Nirvana signifie simplement extinction, souffler et éteindre la soif et la projection de ses désirs, pour laisser apparaitre les phénomènes comme ils sont, dans leur ainséité (juste ainsi, Tathagata, épithète du Bouddha éveillé, signifiant littéralement : ainsi allé, ainsi venu, ou plus exactement selon Bitbol : se tenant dans l'ainsi). C'est ce qui permet à Bitbol d'écrire : «L'état d'absorption, une fois poussé dans ses extrémités, débouche sur un territoire qui n'a rien d'étranger, et qui ne semble insolite que parce qu'il s'est vu dépouiller de son quadrillage cadastral : ce qui est, tel qu'il est ${ }^{48}$. En ce sens, il s'agit juste d'un éveil à ce qui se présente à chaque instant, comme si le méditant parvenait à se résorber dans un champ quantique originel et fondamental d'où émergeraient des événements ou apparitions discrètes, qui s'effaceraient aussitôt, comme d'étranges bosons énigmatiques. Pour parler de cet état ultime, la tradition tibétaine parlera de " présence ouverte », et la tradition zen du retour à un état originellement éveillé et apaisé de la conscience, conscience hishiryo de la non-pensée, avant toute pensé, où la frontière entre le moi et le monde cesse, pour retrouver en soi ce qui est également parfois improprement appelé le « nature de Bouddha » (nature de ce qui est simplement, ainsi).

Notre réflexion débouche ainsi sur un paradoxe étrange : comment concilier la métaphore hindoue de la goutte d'eau qui se fond dans un océan infini d'énergie, avec l'image bouddhiste du miroir vide de toute poussière, qui n'a plus rien à refléter ? Peut-être faut-il accepter l'aporie comme l'endroit et l'envers d'une même réalité, et se dire que l'état mystique ultime n'est finalement rien d'autre qu'un retour à l'état fondamental « apaisé » de l'homme, c'est à dire l'état fondamental au sein de la structure hyperfine de l'état quotidien tranquille. Tel serait le point commun entre « le demi-silence bouddhique

46 Michel Bitbol, La consciende a-t-elle une origine ?, id, p. 181.

47 Heinrich Zimmer, Les philosophies de l'Inde, Joseph Campbell et Payot, 1953, p. 342.

48 Michel Bitbol, La conscience a-t-elle une origine ?, id, p. 180. 
et le verbe théologique : ils cherchent l'un comme l'autre à préparer l'esprit à rencontrer l'inouï et l'innommé en eux-mêmes, l'évoquant dans un cas comme une proto-nature, et le peignant dans l'autre cas sous les traits d'une sur-nature. " ${ }^{49}$. Par rapport à nos hypothèses quantiques, cela impliquerait que le saut quantique induisant un changement d'état de conscience se diluant dans ce qu'on nomme métaphoriquement un océan d'énergie, serait corrélatif ou suivi par un retour de la conscience ordinaire à ce que nous avons nommé l'état fondamental apaisé, par lâcher prise et extinction des préhensions ou crispations de l'ego...

\section{Passage d'un état à l'autre et physique quantique}

A l'issue de cette présentation, les états méditatifs semblent bien répondre à des échelles d'intensité différente, tout comme à un moindre degré les états affectifs et émotionnels de l'esprit humain qui, rappelons-le, peuvent sous le coup d'une excitation accrue, passer d'un état faible, à un état moyen, puis exacerbé - de la contrariété, à la colère et à la rage par exemple. Mais si, pour les états émotionnels de Plutchik, on comprend que le stimulus externe fasse soudain passer d'un état à l'autre, comment se produit le passage autrement plus fort d'un état de méditation $\mid 0>$, à $\mid 1>$, $\mid 2>$, voire $\mid 3>$, alors que le méditant cherche à s'abstraire de tout stimulus extérieur ? Nous allons tenter d'aborder cette question sous trois aspects : celui de la dynamique des relations, des bosons, et enfin des échanges thermiques qui, chacun, permettent de vérifier si les changements produits en méditation répondent bien aux exigences de la physique quantique.

\section{Quelle dynamique des relations, rupture de niveaux ?}

Dans cette deuxième partie, il s'agit donc de se demander comment le passage d'un stade à l'autre peut s'expliquer. On sait qu'au cours de la méditation, les pensées surgissent et disparaissent. Tout comme les bosons d'interaction, la lumière, le photon pour le champ électromagnétique. Les pensées sont-elles des bosons qui émergeraient ponctuellement du champ quantique d'une conscience recentrée sur elle-même et apaisée ? Cette hypothèse mérite probablement des développements futurs ! On peut imaginer aussi qu'elles sont le résultat d'une mesure faite par notre conscience, la trace d'une réduction du paquet d'ondes, la trace de l'interaction entre l'observateur macroscopique qu'est notre conscience et les phénomènes microscopiques de notre corps, notre cerveau en particulier.

Rappelons que nous avons identifié trois échelles différentes dans les niveaux d'énergie qui structurent le psychisme humain. Nous partons de l'état quotidien agité, de l'état psychique ordinaire de la vie de tous les jours, des échanges sociaux. Avec un apport d'énergie important, on passe à l'état amoureux, puis au-delà à l'état mystique qui permet de déboucher à son tour vers un état de contemplation. L'état « quotidien agité » est composé à son tour d'une myriade de sous-états, structure fine du système énergétique. Parmi ceux-ci, la joie, la colère, etc. autant de niveaux d'excitations du quotidien ordinaire. L'« état tranquille » est pour nous le fondamental de ce nouveau système spectral de structure fine. L'état tranquille est l'état de référence du méditant qui descend petit à petit vers des sous-niveaux «hyperfins » pour aboutir à un nouvel état fondamental apaisé, qu'on peut nommer " état de vide », en analogie avec le vide d'un système de champ électromagnétique sans photon. On a donc trois structures emboîtées pour le système psychique, avec des niveaux d'échange d'énergie au sein de chaque sous-système du même ordre, mais des ordres de grandeur très différents entre deux sous-systèmes.

Comme toujours en mécanique quantique, la relation entre les niveaux d'énergie d'effectue par des sauts discrets, des transferts avec une énergie bien déterminée, comme par exemple l'effet photoélectrique introduit avec la relation (1) au début de cette contribution. Dans ce cas, le médiateur de l'interaction, le « boson » comme le nomment les physiciens, est le photon, grain de lumière insécable

49 Idem, p. 170. 
prévu dès 1905 par Albert Einstein ${ }^{50}$. Afin de prolonger l'analogie entre système atomique et psychisme humain, développer notre tentative de spectroscopie du psychisme humain, nous devons partir à la recherche de ce boson d'interaction entre les niveaux d'énergie du psychisme. On sait que la quête d'un boson est toujours une "grande affaire " en physique. Ainsi la compréhension de l'interaction faible avec le modèle de Weinberg-Salam ${ }^{51}$ a permis d'émettre l'hypothèse du « boson intermédiaire », mis en évidence au CERN en $1984^{52}$. Plus récemment, l'unification de l'interaction électro-faible avec l'interaction forte qui lie les protons et les neutrons au sein du noyau atomique a permis la découverte ${ }^{53}$ d'un boson prévu dès 1964 par Robert Brout, François Englert, Peter Higgs ${ }^{54}$ et probablement beaucoup d'autres !

\section{Un boson pour la méditation?}

En physique quantique, le changement de niveau d'énergie pour le système est possible s'il échange un quantum d'énergie avec le monde extérieur. Un photon, grain élémentaire de lumière, permet la transition entre l'énergie E0 et l'énergie E1 si (et seulement si !) sa fréquence $v$ est exactement accordée aux deux énergies précédentes grâce à la relation (1). De façon réciproque, quand l'atome redescend du niveau E1 au niveau E0, il émet un photon de fréquence $v$. Le photon est le boson d'interaction, le médiateur de l'échange entre l'atome et le monde extérieur.

La question maintenant, dans le cadre d'un modèle quantique pour l'Homme, est de comprendre quels sont ces grains d'énergie, ces bosons d'interaction qui permettent par exemple de passer de l'état ordinaire à l'état amoureux. Cette transition se fait de façon brutale en général ; c'est le fameux « coup de foudre ", dont le nom rappelle l'échange de lumière au sein du système atomique. Mais il s'agit également de comprendre ce que sont ces micro-échanges d'énergie qui permettent au médiateur d'explorer la structure hyperfine du spectre humain, de descendre petit à petit vers le niveau fondamental, nommé «vide » dans le cas du système obtenu en quantifiant l'ensemble du champ électromagnétique. Dans le cas de la méditation, l’inversion du regard de l'extérieur du monde vers l'être intérieur permet cette exploration progressive. Mais ce n'est probablement qu'un cadre global de maintien dans un «état tranquille » qui évite les perturbations plus fortes de la vie quotidienne. Le passage précis d'un niveau d'énergie au suivant doit se faire (si le cadre quantique de ce modèle est correct) via une dépense d’énergie, très faible, du sujet. Par contre, la perturbation du monde extérieur

50 Albert Einstein, « Zur Elektrodynamik bewegter Körper », Annalen der Physik. Vol. 17, p. 891-921, 1905.

51 Sheldon L. Glashow, « Partial Symmetries of Weak Interactions », Nuclear Physics, vol. 22, p. 579-588, 1961, Steven Weinberg, « A model of Leptons », Physical Review Letters, vol. 19, issue 21, p. 1264-1266, 1967, Abdus Salam et John C. Ward, « Electromagnetic and weak interactions », Physics Letters vol. 13, issue 2, p. 168, 1966.

52 Simon van der Meer, "Stochastic cooling in the CERN Antiproton Accumulator », IEEE Transactions on Nuclear Science, vol. 28, p. 1994-1998, june 1981, Simon van der Meer, « Stochastic cooling and the accumulation of antiprotons », Review of Modern Physics, vol. 57, issue 3, p. 689-697, 1985, et Carlo Rubbia, " Physics results of the UA1 collaboration at the CERN proton-antiproton collider », Neutrino Physics and Astrophysics, Proceedings of the 11th International Conference held 11-16 June, 1984 at Nordkirchen near Dortmund. Edited by K. Kleinknecht and E.A. Paschos. H. Yuta. Singapore: World Scientific, p. 1, 1984.

53 CERN, annonce de la découverte du boson « BEH » lors d'une conférence de presse sur les expériences Atlas et CMS, 4 juillet 2012, http://home.web.cern.ch/fr/about/physics/search-higgs-boson.

54 François Englert et Robert Brout, «Broken Symmetry and the Mass of Gauge Vector Mesons », Physical Review Letters, vol. 13, issue 9, p. 321-323, 1964, Peter W. Higgs, « Broken Symmetries and the Masses of Gauge Bosons », Physical Review Letters, vol. 13, issue 16, p. 508-509, 1964, Gerald Guralnik, Carl RichardHagen, Thom Kibble, «Global Conservation Laws and Massless Particles », Physical Review Letters, vol. 13, issue 20, p. 585-587, 1964. On pourra lire par exemple le livre de et Gilles CohenTannoudji et Michel Spiro Le boson et le chapeau mexicain : un nouveau grand récit de l'univers, Gallimard, Paris, 2013. 
(mieux : une perturbation issue du monde extérieur ?) se traduit par un apport toujours possible d'énergie qui déplace le méditant d’un niveau d'énergie vers un niveau plus agité.

Quelle est la nature de cet apport d'énergie ? Rappelons d'abord qu'elle doit être de fréquence exactement accordée, comme pour le photon, grâce à une relation de compatibilité analogue à la relation (1). Si le monde extérieur envoie des grains d'énergie désaccordés, même de fréquence beaucoup plus grande, le système ne change pas d'état. C'est une grande découverte d'Albert Einstein en 1905 lorsqu'il fit l'hypothèse du photon. Compte tenu de la structure très complexe de l'être humain, nous pensons qu'il ne faut pas chercher cet échange d'énergie dans l'électromagnétisme pur comme pour les atomes, mais par un assemblage probablement à mettre en évidence de structures biomoléculaires qui organisent in fine les échanges au sein du psychisme humain.

On peut émettre l'hypothèse ${ }^{55}$ qu'un coup de foudre résulte de la mise en résonance entre un souvenir inconscient enfoui dans la mémoire et un moment du présent, avec un travail d'incubation et d'actualisation préalable du souvenir enfoui. Alors cet échange d’information, dont le sujet en général n'a pas conscience, provoque le coup de foudre, le passage de « l'état quotidien » à l'état amoureux. Est-ce la pensée qui réalise cet échange d'information ? Il nous semble clair que penser demande une dépense d'énergie ${ }^{56}$ et se traduit par des échanges d'information au sein du système bio-physique du corps. Mais il est probable que l'accord résonant de la relation (1) n'est pas atteint et que la pensée ne permet pas une telle transition. Au contraire, il semble, si on se réfère à l'hypothèse faite pour le coup de foudre lors de l'apparition de l'état amoureux, que la transition entre deux états échappe à la conscience du sujet. On ne choisit pas de tomber amoureux et on ne choisit pas de ne plus être amoureux. On doit donc probablement, chercher les échanges d'énergie entre les états de conscience, niveaux d'énergie hyperfins du sujet analogues à ceux de l'atome, au sein d'un processus non conscient. Bien entendu, ce processus est favorisé par la pensée qui contrôle l'état psychique conscient ; mais la transition elle-même échappe à la pensée. On peut faire l'hypothèse qu'elle se produit par un échange de boson. Boson qui demande à être mis en évidence. Boson qui permet la transition entre deux états de conscience. Boson émis de façon inconsciente par le système biophysique du corps humain. Boson universel qui ne dépendrait pas de l'individu : tout pourrait ainsi s'expliquer en renvoyant à un niveau biologique à un neurotransmetteur qui serait un boson d'interaction, pas fondamentalement différent de ce qui se passe quand on est amoureux, qui déclencherait et serait accordé au changement d'état qui nous intéresse.

L'éclairage de ce qui se passe dans les changements d'état au cours de la méditation permet d'amener d'autres éclairages complémentaires, en retrouvant de manière étonnante ce qui se passe pour l'état amoureux. Pour qu'un changement d'état permette de quitter l'état quotidien agité, il faut en effet au moins trois conditions : d'abord, nous l'avons longuement vu avec Varela, une suspension du rapport engagé au monde et une redirection du regard vers la vie pré-réflexive avons-nous vu; ensuite, l'installation de ce que nous avons nommé un état tranquille, notamment grâce aux techniques de concentration sur le souffle qui permettent d'installer un minimum de stabilité au sein des apparitions/disparitions discontinues d'événements et excitations diverses; enfin, un contrôle indispensable de l'attention, qui sans relâche ramène la pensée intentionnelle prête à s'échapper vers l'extérieur, à l'intérieur, pour se transformer en simple présence attentive et intuitive, ouverte et disponible à ce qui se passe à chaque instant. Ce rôle important, voire déterminant de l'attention comme troisième facteur, est confirmé par les études en neurosciences qui mettent en exergue l'importance de la mobilisation du cortex préfrontal, siège entre autres de la régulation de la pensée par l'attention. Mais ce qui est intéressant, c'est que ces trois facteurs sont des conditions nécessaires mais non suffisantes pour qu'un changement d'état important se produise en méditation. Ni la redirection introspective du regard, ni l'installation d'un état de tranquillité, ni l'ouverture d'une attention large sous forme d'accueil et acceptation sans borne à $360^{\circ}$ comme dit Bitbol, ne suffisent à

55 Dans la lignée de Théodule Ribot : Esai sur les passions, Alcan, Paris, 1910.

56 Voir par exemple Giuseppe Vitiello, « Dissipation and memory capacity in the quantum brain model », International Journal of Modern Physics B, vol. 09, issue 08, p. 973-990, 1995. 
expliquer ou provoquer un changement d'état, de l'état quotidien agité vers un état mystique, ou de vide et d'accueil : ce changement ne sera jamais déterminé et provoqué causalement, il apparaitra la plupart du temps de manière soudaine et discontinue, voire brutale, comme dans l'état amoureux.

Dans le Zen Soto, il est dit qu'on peut juste favoriser l'éclosion de l'éveil, mais en aucun cas le provoquer : il se produira spontanément, naturellement, automatiquement et inconsciemment comme aimait bien le dire Deshimaru ${ }^{57}$, sans que l'homme y intervienne en quoi que ce soit. Sous l'effet biologique d'un neurotransmetteur, d'une relation soudaine de compatibilité, ou pour reprendre la terminologie zen lorsque, mystérieusement, le corps, le mental et la respiration se retrouvent enfin synchronisés et réunifiés dans une posture " juste », en phase : à ce moment, en même temps que le « lâcher prise », une illumination soudaine peut se produire.

Le Zen Rinzaï sera encore plus radical, en prônant non une illumination progressive qu'on peut préparer comme dans le Zen Soto, mais une illumination subite. Pai-Tchang Houai-Hai, maître Tch'an chinois du huitième siècle (le Tch'an étant la forme chinoise originelle qui donnera naissance au zen japonais), répondait ainsi à un disciple qui lui demandait comment atteindre la délivrance: « On ne peut l'atteindre que par l'illumination subite " ${ }^{58}$. D'où le développement ultérieur des koans, ces devinettes apparemment absurdes que les maîtres proposent à leur disciple. Lorsque l'élève laisse enfin tomber ses efforts intellectuels pour le comprendre, peut se produire une illumination soudaine, satori, qui le fait accéder à un autre niveau de réalité ou de conscience. "Satori est une expérience spirituelle ; elle décrit le foudroyant déclic de l'illumination bouddhiste. Si celle-ci est difficile à décrire pour un non initié, l'approche intellectuelle est facilement accessible. Il suffit d'imaginer Archimède dans sa baignoire qui découvre la fameuse poussée du même nom: "Eureka ! ». Il expérience un processus cognitif dont la violence s'apparente à une illumination. Tout à coup, l'incompréhensible s'illumine devant l'éclair de l'esprit. C'est un processus soudain, dont l'instantanéité contraste avec la lourdeur d'une explication verbale ${ }^{59}$.

Dans la tradition hindouiste et Vedanta, l'accès aux samadhi et aux niveaux ultimes de conscience ne peut de la même manière se produire que soudainement, avec une rupture ontologique brutale de niveau de conscience, comme le note Mircea Eliade : « Dans le samaddhi a lieu la rupture de niveau que l'Inde vise à réaliser, et qui est le passage paradoxal du connaître à l'être... Cette enstase constitue en fait un rapt, puisqu'elle est éprouvée sans qu'on la provoque... L'enstase équivaut à une réintégration des différentes modalités du réel en une seule modalité : la non dualité primordiale, la plénitude non différenciée d'avant la bipartition du réel en sujet-objet... (avec le sentiment de) l'unité et la béatitude. Il y a retour à l'origine, mais enrichie des dimensions de la liberté et de la transconscience (ou sur-conscience) ${ }^{60}$.

\section{Changements d'état et changements thermiques.}

Lors de la méditation, le sujet navigue au sein des structures hyperfines de l'état tranquille. On a envie de faire la statistique relative à l'occupation de chacun des niveaux d'énergie. Mais on ne peut pas parler de température car on étudie a priori un seul sujet. Toutefois, on peut imaginer faire des moyennes temporelles à partir du temps que le sujet passe dans chaque état. Il s'agit d'une approche un peu osée car on a une vraie dynamique de changement d'état au cours du temps. Or on tente de la décrire avec les outils statistiques de la thermostatique, qui suppose une invariance dans le temps de l'ensemble de l'expérience. Tentons toutefois de poursuivre cette analogie. La population $\mathrm{n}_{\mathrm{j}}$ du niveau $\varepsilon_{\mathrm{j}}$ de la physique statistique représente le temps $\theta_{\mathrm{j}}$ passé dans l'état d'énergie Ej. Alors on peut grâce à

57 Taisen Deshimaru, Zen et arts martiaux, Albin Michel, Paris, 2000.

58 Hoai-Haï, Po-Chang Kouang-Lou, in Hermes n²4, Tch'an et Zen, Fayard, 1985.

59 Ombres nippones, abécédaire, in kichigai.com

60 Mircea Eliade : Histoire des croyances et des idées religieuses, tome 2, Payot, 1978, p. 70 
la fonction de partition « $\mathrm{Z}{ }^{61}$ définir une « température » du méditant, modulo cette transformation qui consiste à considérer la population $n_{\mathrm{j}}$ de l'état pendant le temps $\theta_{\mathrm{j}}$ :

$$
\mathrm{Z}=\Sigma_{\mathrm{j}} \exp \left(-\varepsilon_{\mathrm{j}} / \mathrm{kT}\right) ; \quad \mathrm{n}_{\mathrm{j}}=\exp \left(-\varepsilon_{\mathrm{j}} / \mathrm{kT}\right) / \mathrm{Z} \text {. }
$$

On observe alors sur la relation (2) que la température psychique est négative si le sujet passe plus de temps dans les états excités que dans l'état fondamental.

Dans les méditations, cela impliquerait également que lorsqu'on passe de l'état quotidien agité à l'état tranquille, il devrait y avoir une diminution de température et une régulation des fonctions vitales. Or c'est très exactement ce qu'on constate puisque les études en neurosciences sur le sujet montrent que la méditation provoque quasiment mécaniquement une diminution de la libération du cortisol du stress, une régulation de la pression artérielle et du rythme cardiaque, une élévation des défenses immunitaires $^{62}$, et pour certains types de méditation, une diminution de la température de la peau ${ }^{63}$ !

\section{Conclusion}

En conclusion, si nous reprenons l'hypothèse fractaquantique à maxima, l'analogie constatée entre le système atomique et le système psychique peut se poursuivre : de même que le photon permet de passer d'un état à l'autre du système atomique, de même il devrait exister un boson (qui reste à identifier!) qui serait la cause directe de la transition entre deux états psychiques. Ce boson pourrait être de nature physique, biophysique, chimique ou purement électromagnétique.

Sans aller jusqu'à une telle prédiction, l'hypothèse fractaquantique peut minimalement remarquer analogiquement l'existence d'un même mécanisme et processus expliquant les changements d'état au niveau quantique et au niveau psychique notamment de la méditation. La méditation, lorsqu'elle parvient en effet à un état d'attention stable et apaisé, permettrait ainsi d'aborder ou d'expérimenter un champ quantique sous-jacent de la conscience vide de tout contenu, lequel se manifeste localement et ponctuellement par de petits sursauts ou quantums d'énergie, fragments de pensées, d'émotions ou de sensations fugaces apparaissant puis disparaissant aussitôt, en pouvant laisser ultimement dans un état de joie et de présence apaisée ${ }^{64}$. Comme si ces modifications et ces modulations infimes vécues pendant la méditation, étaient des notes de musique reflétant un champ quantique fondamental, un chant quantique, un cantique de joie...

François Dubois et Christian Miquel, 9 juillet 201465.

61 Voir par exemple le livre de Bernard Diu, Claudine Guthmann, Danielle Lederer et Bernard Roulet : Physique Statistique, Herrmann, Paris, 1997.

62 Voir A. Lutz : « Le cerveau méditatif », Cerveau et psycho numéro 52, 2012 ;

63 Voir R. Manocha, D. Black, D. Spiro, J. Ryan, C. Stough. Changing Definitions of Meditation- Is there a Physiological Corollary? Skin temperature changes of a mental silence orientated form of meditation compared to rest, Journal of the International Society of Life Information Sciences, vol. 28, issue 1, p. 23-31, march 2010. D’autres méditations tibétaines ou de yoga peuvent au contraire viser à augmenter la température de la peau pour lutter contre le froid.

64 Nous pensons plus spécifiquement au deuxième et troisième stade des méditations dhyana et samaddhi précédemment citées. Pour plus de détails sur la manière dont la méditation peut aboutir finalement au retour à un instant présent pleinement ouvert, dans une forme de connaissance paradoxale que nous avons appellée la docte ignorance, c.f. Christian Miquel : Apologie de l'instant et de la docte ignorance, L'Harmattan, 2014.

65 Echanges à Trouville les 20 et 21 mai 2013, mise en forme à Versailles le 22 novembre 2013, travail en commun les 23 et 24 novembre 2013 à Trouville, modification du titre suite a l'envoi au colloque de l'UES fin décembre 2013, nouvelle version suite aux séances de travail les 7 mai et 9 juillet 2014 à Issy les Moulineaux. 
